\title{
DEFINIZIONE DELLA COMPOSIZIONE DEI CAMPIONI DI CERAMICA CON IL METODO DELL'ATTIVAZIONE NEUTRONICA
}

\author{
MÁRTA BALLA \\ Università di Scienze Tecniche e Economiche di Budapest, Cattedra di Tecnologia Nucleare \\ Müegyetem rakpart 9, H-1111 Budapest, Ungheria \\ E-mail: balla@reak.bme.hu
}

\begin{abstract}
Analysis of the composition of pottery with neutron activation. Determining the provenance. Based on the results of the examination of 55 samples, the vast majority of the analyzed pottery originated from the same place, they were probably local products. Some slightly different pieces were identified as well, which was perhaps due to a difference in their dating or in the production technology. Six potsherds proved to be significantly different concerning their chemical composition. These were supposedly imported vessels.
\end{abstract}

Keywords: coarse pottery, determining the provenance, chemical composition, import vessels

Similmente ai risultati ottenuti nel corso delle analisi precedenti (BME-NTI/USTEB-CTN - 625/2013) anche ultimamente l'analisi delle ceramiche ha avuto come risultato una forte somiglianza chimica, e un quadro complessivo degli elementi omogeneo. Secondo la composizione chimica, dunque, i singoli pezzi, salvo qualche rara eccezione, provengono dallo stesso luogo.

I dati ottenuti sono stati sottoposti a algoritmi con molte variabili. La Fig. 1. rappresenta il risultato dell'analisi dei 25 campioni, finalizzata alla definizione del Componente Principale. Stando ai calcoli i campioni n. 20 e 16 sono sicuramente da ritenere di provenienza diversa. Dal gruppo principale si distinguono leggermente anche i campioni n. 1, 5 e 23, ma in questo caso le differenze sono meno significanti.

La Fig. 2. rappresenta l'elaborazione comune dei campioni analizzati nel 2013 e di quelli indagati di recente, entro l'ambito del presente saggio. (la lettera $U$ che precede i numeri distingue i campioni attuali da quelli analizzati in passato)

Analizzando nel 2013 il contesto di reperti in questione si è potuto stabilire con assoluta sicurezza che la provenienza dei campioni n. 6, 26 e 28 era diversa dagli altri. Tra gli oggetti indagati ultimamente abbiamo trovato la maggior dissomiglianza nel caso dei frammenti n. 16 e 20, e come l'anno passato, anche questa volta la spiegazione della composizione chimica diversa dei campioni 6 e 28 va cercata nell'altissimo contenuto di terre rare e ciò caratterizza anche il campione di quest'anno, segnato con il n. U20. Il campione di prima, segnato con il n. 26 si distingueva dal gruppo di campioni omogenei per il contenuto di $\mathrm{Rb}$ e Cs e lo stesso è vero anche per il campione U16.

Nel 1998 avevamo già eseguito la misurazione dei frammenti di ceramica ritrovati in una cisterna scavata. La Fig. 3.

In base ai risultati si può costatare che la maggior parte delle ceramiche analizzate proviene dallo stesso luogo, quindi si tratta probabilmente di merce locale. Sono presenti però anche frammenti che mostrano lievi dif- 


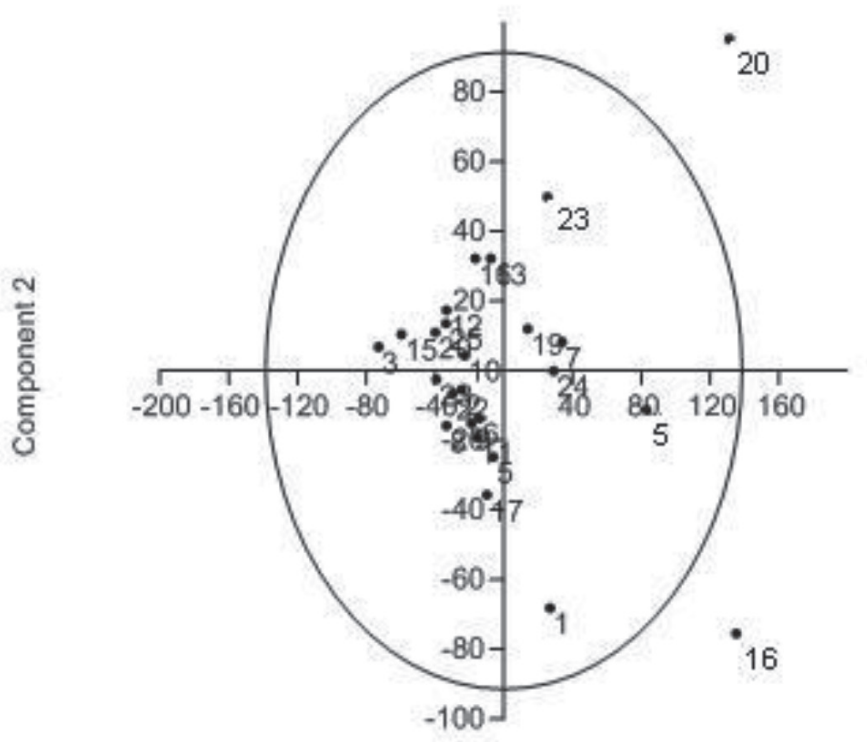

Component 1

Fig. 1.

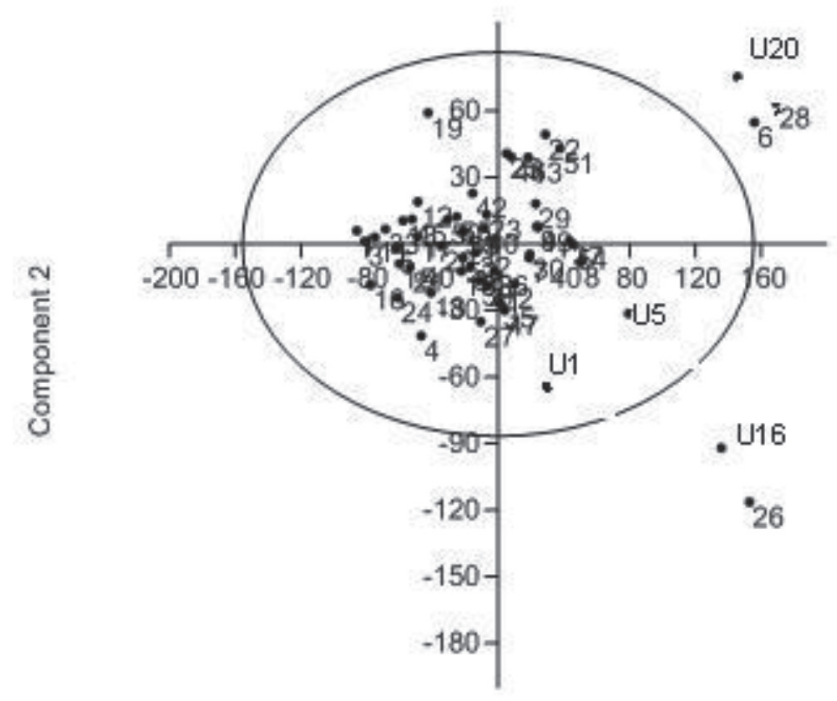

Component 1

Fig. 2. 


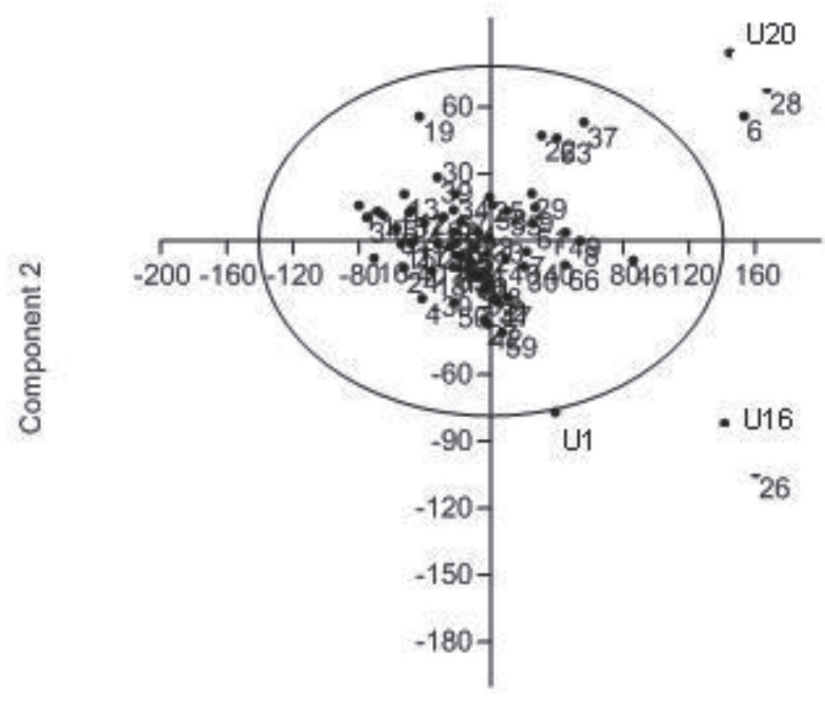

Component 1

Fig. 3 .

ferenze forse di età o di tecnica della produzione, mentre sei campioni rivelano differenze di natura chimica di gran rilievo che permettono di ipotizzare che derivassero da importazione.

\section{VALUTAZIONE DELLE ANALISI DI ATTIVAZIONE NEUTRONICA}

\section{Dénes Gabler}

Negli anni 2013 e 2014 abbiamo incaricato la Cattedra di Tecnologia Nucleare dell’Università di Scienze Tecniche ed Economiche di Budapest di analizzare 55 campioni di ceramica in tutto. La distribuzione secondo i siti di ritrovamento dei campioni di ceramica consegnati ai fini di analisi è la seguente:

cisterna scavata nel taglio g-4

11 campioni in tutto

$\begin{array}{llll}\text { di cui } & \text { brocche } & 1 & \text { campione } \mathrm{n}^{\circ} .25 \\ & \text { vasi potori } & 3 & 27,30, \mathrm{U} \mathrm{19} \\ \text { pentole } & 4 & \mathrm{U} 17, \mathrm{U} \mathrm{18}, 28,29 \\ & \text { olle } & 3 & 26, \mathrm{U} \mathrm{15}, \mathrm{U} 16\end{array}$

Tra i campioni c'era anche una copia di ottima qualità di una terra sigillata con forma Consp. 34

cisterna e fogna nel centro del cortile settentrionale

24 campioni in tutto

$\begin{array}{llll}\text { di cui } & \text { brocche } & 5 & 13,15,22,23, \text { U } 22 \\ & \text { vasi potori } & 3 & 9,10,14 \\ & \text { pentole } & 5 & 16,17,24,21, \text { U } 24\end{array}$




$\begin{array}{lll}\text { olle } & 7 & 6,7,11,12,19,20, \text { U } 23 \\ \text { bacini } & 2 & 1,3 \\ \text { coperchi } & 2 & 8,18\end{array}$

Del bacino, essendo questo di grandi dimensioni, abbiamo prelevato due campioni; nel dendogramma pubblicato da $\mathrm{M}$. Balla (i campioni $\mathrm{n}^{\circ} 1$ e 3 ) che sono quelli che si trovano più vicini uno all'altro.

strato d'incendio ubicato nella parte $\mathrm{S}$ della prima villa

17 campioni in tutto

di cui

$\begin{array}{lll}\text { brocche } & 1 & \\ \text { vasi potori } & 1 & 5 \\ \text { pentole } & 5 & 4,\end{array}$

$\mathrm{U} 13$

olle $\quad 7$

4, U 2, U 5, U 11, U 12

tipo ? $\quad 3 \quad \mathrm{U} 10, \mathrm{U} 14, \mathrm{U} 4$ fuori contesto

3 campioni in tutto

di cui ceramica a pareti sottili $2 \quad$ U 20, U 21

olle $\quad 1 \quad$ U 25

Nel corso delle analisi, dunque, siamo venuti a sapere che, il vasellame facente parte dei cinque gruppi individuati durante le ricerche petrografiche probabilmente vennero fabbricati in una data officina o, al limite, nella stessa area di officine, nonostante che, tra il riempimento della cisterna scavata nel taglio g-4 e quello della cisterna e fogna rinvenuto nel cortile settentrionale siano passati ben 200 anni. Si ipotizza quindi, che il vaso a forma di Consp.34, di qualità simile a quella delle sigillate, dovette essere fabbricato nello stesso luogo dove fu fatta la ceramica comune di cui sopra.

Tra i 6 esemplari che differiscono in modo rilevante dagli altri:

dalla cisterna del taglio g-4 sono venute alla luce 2 pentole

dalla cisterna del cortile $\mathrm{N} \quad 2$ olle

dallo strato d'incendio 1 olla

fuori contesto (taglio $\mathrm{d}+2$, nelle vicinanze del canale che porta fuori dall'edificio):

2 ceramiche a pareti sottili

I fatti summenzionati attestano la nostra ipotesi secondo la quale la cisterna e fogna attigua, ubicati nel cortile settentrionale furono riempiti alla fine del III secolo con terriccio contenente frammenti di ceramica provenienti dalla seconda metà del I secolo e dalla prima metà del II secolo. L'officina o le officine locali, quindi producevano vasellame da tavola e anche vasi di cucina di ceramica e questa pratica duró un periodo relativamente lungo.

La quantità della merce importata era limitata, avrà superato appena il $10 \%$. Gli altri oggetti di ceramica probabilmente vennero fabbricati altrove, in altre officine dell'Italia Centrale, e questo materiale contiene anche ceramiche a pareti sottili. 